\title{
Guest editorial: special issue on nature inspired cooperative strategies for optimization
}

\author{
Rodica Ioana Lung · Camelia Chira · D. Dumitrescu
}

Published online: 2 November 2012

(C) Springer-Verlag Berlin Heidelberg 2012

Nature inspired computing is nowadays part of a global emerging paradigm. Diverse biological processes, natural evolution and other complex cooperative systems in nature have always been a fruitful source of inspiration for computer science leading to the development of highly effective problem solving algorithms and computing strategies. Well known examples include evolutionary algorithms, ant colony optimization, artificial immune systems, particle swarm optimization, membrane computing and artificial bee colonies.

The current special issue contains the extended versions of the best research papers presented at the $\mathrm{V}$ International Workshop on Nature Inspired Cooperative Strategies for Optimization (NICSO) held on October 2011 in Cluj-Napoca, Romania. The aim of NICSO is to provide an inspiring environment for debating the state of the art ideas and techniques in nature inspired cooperative strategies and their most recent applications. The topics covered by NICSO 2011 include swarm intelligence, genetic algorithms, multiagent systems, coevolution and cooperation strategies, adversarial models, synergic building blocks, complex networks, social impact models, evolutionary design, self organized criticality, evolving systems, cellular automata, hybrid algorithms and membrane computing.

The papers included in this collection contain research results of new swarm intelligence models, genetic and

\section{R. I. Lung $(\varangle)$}

Faculty of Economics and Business Administration,

Babeş-Bolyai University, Cluj-Napoca, Romania

e-mail: rodica.lung@econ.ubbcluj.ro

C. Chira · D. Dumitrescu

Department of Computer Science, Babeş-Bolyai University, Cluj-Napoca, Romania

e-mail: cchira@cs.ubbcluj.ro

D. Dumitrescu

e-mail: ddumitr@cs.ubbcluj.ro hybrid algorithms, differential evolution and cooperative co-evolution algorithms in addressing various optimization problems.

The work of M. Majid al-Rifaie, J.M. Bishop and T. Blackwell integrates a nature inspired swarm intelligence algorithm with a biologically inspired evolutionary algorithm by applying the resource allocation mechanism deployed in stochastic diffusion search to the differential evolution. Numerical results reported show a better performance of the proposed hybrid algorithm compared to standard differential evolution for several benchmarks.

A. Lihu, O.-A. Popescu and S. Holban present a composite mutation operator for real-valued genetic algorithms. The evolutionary process is refined by using a Gaussian schemebased disagreements operator. Experiments conducted for several optimization problems confirm the potential of the disagreement mutation operator particularly for genetic algorithms with small population size.

The work of H. Sharma, J.C. Bansal and K.V. Arya extends the well-known differential evolution method by introducing a fitness based position update process inspired by the artificial bee colony algorithm. The efficiency of the proposed method is studied in a set of extensive experiments for 22 benchmark optimization problems and for the model order reduction problem.

M.R. Karim and C. Ryan present an Attribute Grammar with Lookahead (AG+LA) approach that incorporates a form of lookahead into the mapping process of Grammatical Evolution (GE) using Attribute Grammar (AG) and also an improved version $\mathrm{AG}+\mathrm{LAE}$ that endows $\mathrm{AG}+\mathrm{LA}$ with an efficiency measure to bias the search towards the most efficient (best) objects.

A.P. Tonda, E. Lutton and G. Squillero present a benchmark problem for the study of cooperative co-evolution algorithms. The problem models a search for the optimal 
placement of a set of lamps in a room which leads to a fitness function that is a trade-off between conflicting objectives. The performance of three cooperative strategies, Parisian Evolution, Group Evolution and Allopatric Group Evolution is evaluated using the proposed lamp benchmark.

The paper by J.R. Villar, S. Gonzalez, J. Sedano, E. Corchado, L. Puigpinos and J. de Ciurana focuses on feature selection for a real-world application, steel sheet incremental cold shaping process, by dealing with open issues such as analyzing the relevance of the different crossvalidation methods, the reduction of the parameter setting for the method and the relevance of including auto-tuning methods.
The contributions presented in this issue form a high quality research material that will hopefully enrich the field of cooperative strategies for optimization and promote further developments in this area. As guest editors, we would like to thank the authors for their highly valued contributions, the reviewers for their excellent work in assessing the Scientific quality of the submissions and the editors of the Journal of Memetic Computing for their support and patience. C. Chira and R.I. Lung acknowledge the support from grant PN II TE 320, "Emergence, auto-organization and evolution: New computational models in the study of complex systems", funded by CNCS Romania. 